\title{
DESCRIPTION OF A MODEL OF THE GYROSCOPE FOR CLASS ILLUSTRATION.
}

\section{By B. Howard Rand, M.D.}

The gyroscope is usually spoken of as a toy. It is not such. It is as important an illustration of the law of centrifugal force as the Atwood machine is of that of gravitation.

It is seldom described in those gatherings of blunders known as "text-books;" and so much the better for the poor children who have to commit the statements (true or false) to memory. It is still more rarely shown to a class, perhaps because the "professor" does not understand it himself.

One great difficulty for the beginner in the study of mechanics is the erroneous definition of inertia; another, the misleading use of the words "tendency" and "tend," as applied to particles under the influence of force.

Inertia in the dictionaries and encyclopodias is used as synonymous with vis inertioe. In the text-books it is described as a property of matter by which when at rest it "tends" to remain at rest, and when in motion to continue in motion in its original direction. That dead matter can exert force, as the term vis inertice requires, is absurd; that matter can "tend," is equally so. The mistake has continued from the fact that the compilers of text-books have copied the errors of their predecessors, and the teacher too often follows the book without independent thought.

Inertia is not a force, neither is it a property of matter. It is a convenient word to represent the fact that all change in matter, whether physical or chemical, is due to force. Matter, as we see it, is composed of many particles, hence the communication of the force requires time, in order to affect each particle in succession. Force is never lost, and a body influenced by it will remain under its influence until the force is neutralized or altered by other forces, either active or so-called passive. Among the latter are classed friction and resistance of the air by which the original force is converted into a new form, usually heat.

Perhaps the best illustration of the fact that the "inertia" of a body at rest is due to the law that the communication of motion to a mass requires time, is to be found in the starting of a long train of coal cars. "The driver backs his engine until the "bumpers" touch 
and the coupling links are slackened; then, starting the engine, it picks up one car after another, and thus puts the train in motion. Were the train "screwed" together, the adhesion of the driving-wheels. would fail to give purchase enough to start it.

The "inertia of the plane of rotation," as seen in a boy's hoop or top, means simply that the original museular force applied has to be exhausted before that of gravity can show its power.

The gyroscope is so well known that a description of it might seem needless. It may be worth the little time necessary to describe it to those who wish to follow the ideas of the model closely.

It is a smooth, heavy disc of metal on a shaft, which is suspended in a ring by two points at its ends, so as to permit of free rotation, Fig. 1. It is started by quickly drawing off a string, previously wound around the shaft after the fashion of a boy's top. The ring is firmly fastened to a rod, which has at its middle a cup, and on the other side of the cup a counterpoise weight, which can be fixed by a set screw so as to balance, underbalance or overbalance the disc. The cup rests on the point of an upright stem fastened in a base board; thus, hung like the ordinary magnetic needle, the whole is free to move in both a horizontal and a vertical plane.

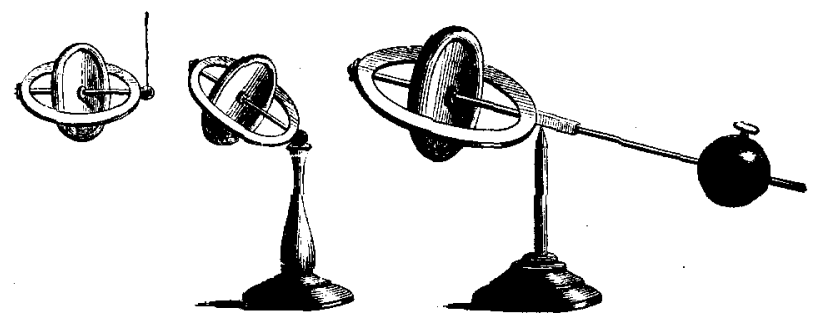

Fig. 1.

When the disc is balanced and the string pulled no movement, other than its own on its axis, is noticed. For convenience, it may be supposed to have right-handed rotation, to wit, that of the hands of a watch.

When the dise is not in rotation, if underbalanced it falls to the base-board or table, if overbalanced it is caused by the weight of the counterpoise to rise. Although these movements are not strictly in a vertical plane, they may, for the purpose of making the explanation shorter, be so considered.

When the disc, after being set whirling, is underbalanced, instead of falling it remains in the vertical plane, and at the same time a motion, 
called "precessional," of the whole system around the upright stem is noticed. This precessional motion is more rapid as the velocity of the disc is greater. It is in an horizontal plane, and its direction is righthanded. Being in a plane at right angles to that of the disc, the motion is in a direction opposite to that of the upper edge of the disc. As the disc gradually, from friction of the bearings and of the air, comes to rest, the precessional motion diminishes, slowly ceases, and the disc falls. When overbalanced these movements are reversed.

The model which I submit is intended to explain these phenomena. It presupposes a knowledge of the principle of the composition and. resolution of force. It can be made by any one of thick pasteboard, or at a small expense by a tinsmith.

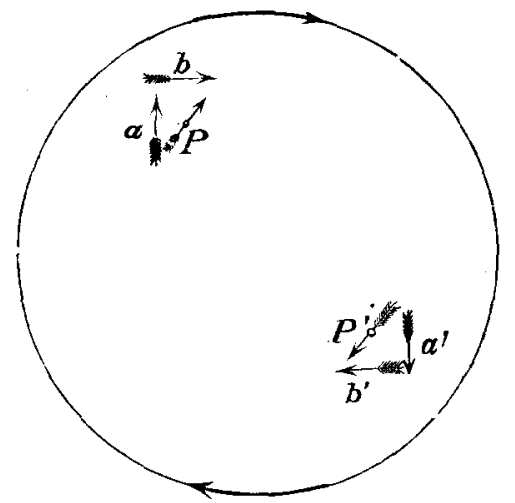

Fig. 2.

$a a^{\prime}$ vertical components. $\quad b b^{\prime}$ horizontal components. $\quad P P^{\prime}$ Particles under influence of the centrifugal force.

On the opposite sides of the disc, which is mounted on a stem with a counterpoise, are cut slots in the form of arrows, as represented in the cut, Fig. 2. In these slots are placed arrows, slightly smaller than the slots. These are hung by wire near the barb, so that they move freely through the slots. The arrow $(a)$ on the left-hand side is weighted by solder at its butt, that on the righthand side $\left(a^{\prime}\right)$ is weighted at its point. When the dise is tilted the arrows will still remain vertical, but with the points in opposite directions. The arrangement of the horizontal arrows will be explained later. The supposed particles, $p$ and $p^{\prime}$, indicated by dots painted on the disc, are under the influence of two forces; first, that of the arm used in drawing off the string. This impels them in the direction given 
when the disc was in a vertical plane. Second, that of gravity, which pulls the disc towards the earth.

We have represented by the arrows the components, $a$ and $b$, and $a^{\prime}$ and $b^{\prime}$ of the resultant motion of the two supposed particles, one near the upper edge of the disc, the other near the lower edge, and on opposite sides; they are moving in opposite directions at the same time. The direction of the rotation is shown by arrows painted on the disc near its upper and lower edges.

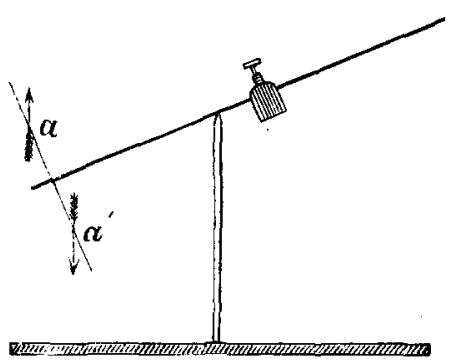

Fig. 3.

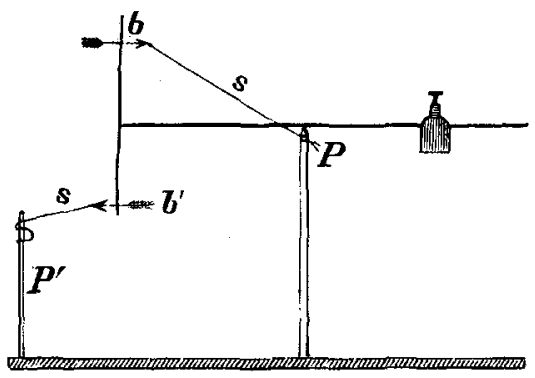

Fig. 4.

When the dise is tilted (Fig. 3) the vertical components remain vertical, like plumb-lines. The pressure of $a$ upwards and $a^{\prime}$ downwards on opposite sides of the disc, due to their continuing in their first direction by virtue of the original force applied, will cause a movement around the point of support, $s$, on the known principle of a "couple," or bent lever. This can readily be shown by pressing at the same time on the front and back of the dise at the points indicated by the barbs of the arrows, the disc being counterpoised. The precessional motion will result.

The arrows representing the horizontal components are hung to the disc by a wire hinge near the point. They are attached by strings, $s$, to posts on the base-board, $P$ and $P^{\prime}$, one in front of the disc, the other behind it. The holes for the strings are in the barbs of the arrows. The heads of the arrows indicate the pressure of the original force in opposition to an attempt to change the direction of the original vertical plane of rotation by the precessional movement. This can be shown by pressing near the upper and lower edges of the dise, as the arrows point, when the disc will be forced upward by a vertical force acting as a couple, or through a bent lever, as before, 
Fig. 4. The neutralization of the force of gravitation acting on the dise is thus shown.

This explanation of the phenomena of the gyroscope is not claimed is original. The plan of the model for class illustration is believed to be so. The model is on deposit at the Franklin Institute, and any one is at liberty to make a copy.

\section{SOUND FROM RADIANT ENERGY.}

The announcement that Mr. W. H. Preece, the President of the Society of Telegraph Engineers, would lecture on the "Photophone and the Conversion of Radiant Energy into Sound," attracted a large number of auditors to the hall of the Institution of Civil Engineers on Wednesday, December 8th. This was partly due to the intrinsic interest of the subject coupled with the well-known ability of the lecturer, and partly to the fact that Professor Bell himself was expected to be present. Mr. Preece began his discourse by a reference to the other three great inventions of the last four years: the telephone, the phonograph, and the microphone, all of which, together with the latest marvel, the photophone itself, were concerned in the transmission and reproduction of sound.

After explaining that sound was a sensation caused by a vibratory motion in the air communicating itself to the drum of the ear, he illustrated the composite nature of light by throwing a splendid spectrum on the screen with the help of Mr. Ladd. Light, he observed, is a sensation of the eye set up by the action of a vibratory motion in the luminiferous ether. The telephone owes its action to an electric current varying in sympathy with the vibrations of the air constituting sound, and it is clear that if a substance could be got which, under the influence of light, would vary an electric current passing through a telephone, the transmission of sound to a distance by means of a vibratory beam would be rendered feasible. Such a substance is selenium, a body discovered in the year 1817 by Berzelius, when he was looking for tellurium, but it is so intractable in its physical properties that Professor Bell has had to overcome a great many practical difficulties in arlapting it to his purpose.

We have so recently described the genesis of the photophone and its artual construction that it will be unnecessary for us to follow Mr. 